PROCEEDINGS OF THE

AMERICAN MATHEMATICAL SOCIETY

Volume 132, Number 1, Pages 183-193

S 0002-9939(03)07015-1

Article electronically published on May 22, 2003

\title{
EXISTENCE OF SOLUTIONS TO PROJECTED DIFFERENTIAL EQUATIONS IN HILBERT SPACES
}

\author{
MONICA-GABRIELA COJOCARU AND LEO B. JONKER \\ (Communicated by Carmen C. Chicone)
}

\begin{abstract}
We prove existence and uniqueness of integral curves to the (discontinuous) vector field that results when a Lipschitz continuous vector field on a Hilbert space of any dimension is projected on a non-empty, closed and convex subset.
\end{abstract}

\section{INTRODUCTION}

In this paper we study the existence of solutions for a class of ordinary differential equations with discontinuous and non-linear right-hand side, the so-called projected differential equations. This class of equations was first introduced in [D-I] and applied to study the dynamics of solutions to finite-dimensional variational inequalities in [D-N], [Na2, [Na3, [Na4, [Na5], [Na6]. In all these works the existence theory for solutions to projected differential equations was formulated on Euclidean space.

Finite-dimensional variational inequalities theory provides solutions to a wide class of equilibrium problems in mathematical economics, optimization, management science, operations research, finance, etc. (see for example $\mathrm{He}$, [s], D-N], [Na1, Na2], [Na5]). Therefore there has been a steady interest over the years in studying the stability of solutions to finite-dimensional variational inequalities (and consequently the stability of equilibria for various problems), as can be seen in [A-C], [He], [Na2], Is-C1], [ss-C2], [P]. In [D-N] and [Na2] this study is done by associating a dynamical system to a variational inequality. This system is given by the solutions to a projected differential equation.

Following the methodology employed in $[\mathrm{D}-\mathrm{N}]$ and $[\mathrm{Na} 2]$ in the finite-dimensional case, we are interested in studying the dynamics of solutions to infinite-dimensional equilibrium problems that can be solved via infinite-dimensional variational inequalities. Examples of this kind of problems can be found among free boundary and optimization problems (the lubrication problem, the obstacle problem, flow through a porous medium-see [B-C] and [K-S]).

In this paper we present the first step in this study: we show that a projected differential equation has solutions on a Hilbert space of any dimension. In brief, if $X$ is a Hilbert space, $K \subset X$ a closed, convex subset, and $F$ a Lipschitz continuous vector field, we study the vector field obtained by projecting $F$, at each $x \in K$, to

Received by the editors June 27, 2002 and, in revised form, September 9, 2002.

2000 Mathematics Subject Classification. Primary 34A12, 34A36; Secondary 34A60, 49J40.

(C)2003 American Mathematical Society 
the tangent cone of $K$. This vector field is discontinuous on the boundary of $K$. We prove the existence and uniqueness of integral curves and show they remain in $K$.

Some of the problems mentioned above (for example flow through a porous medium, see $\mathrm{K}-\mathrm{S}$, Chapter VII) already involve time. However, the variational inequality theory only identifies steady-state solutions for these problems. As in the finite-dimensional case, a dynamics given by solutions to a projected differential equation is interesting because it describes these problems as dynamical systems and has the potential for modelling the return (or not) to steady state when the system has been perturbed.

Our goal in this paper is to present the mathematical technique involved in proving the existence of solutions to projected differential equations in an infinitedimensional setting, since this technique is different from the one used in the finitedimensional case in [D-I], [D-N] and [Na2].

The paper is organized as follows: in Section 2 we outline some preliminary definitions and results, we give the definition of a projected differential equation and show the relationship between a projected differential equation and a variational inequality. In Section 3 we formulate the main result of the paper, Theorem 3.1, and present its proof. We conclude with a few remarks.

\section{Projected Differential EQUations}

Let $X$ be a Hilbert space of arbitrary dimension and let $K \subset X$ be a non-empty, closed and convex subset. For any $z \in X$, there exists a unique element in $K$, denoted by $P_{K}(z)$, such that $\left\|P_{K}(z)-z\right\|=\inf _{y \in K}\|y-z\|$. This defines a mapping $P_{K}: X \rightarrow K$ given by $z \mapsto P_{K}(z)$, called the projection operator of the space $X$ onto the subset $K$. The properties of the projection operator on Hilbert spaces have been studied extensively (see $[\mathrm{Z}]$ ). In particular $P_{K}(y)=y$, for any $y \in K$. At this point we assume that the reader is familiar with the concepts of convex cone, polar cone and set-valued mapping.

Definition 2.1. (i) The set $T_{K}(x)=\overline{\bigcup_{h>0} \frac{1}{h}(K-x)}$ is called the tangent cone to $K$ at the point $x \in K . T_{K}(x)$ is a closed convex cone.

(ii) The normal cone to the set $K$ at the point $x$ is the polar cone of $T_{K}(x)$ and is given by $N_{K}(x):=\left\{p \in X \mid\left\langle p, x-x^{\prime}\right\rangle \geq 0, \forall x^{\prime} \in K\right\}$. The normal cone is a closed, convex cone.

Lemma 2.1. The set-valued mapping $x \mapsto N_{K}(x)$ is monotone; i.e., for any $x \neq$ $y \in K$ and any $n_{x} \in N_{K}(x)$ and $n_{y} \in N_{K}(y)$ it satisfies $\left\langle n_{x}-n_{y}, x-y\right\rangle \geq 0$.

Proof. The conclusion follows immediately from the definition of the sets $N_{K}(x)$ and $N_{K}(y)$.

The following result can be found in [Ra], Theorem 2.23.

Theorem 2.1 (Moreau). If $C \subset X$ is a closed convex cone and $x, y, z \in X$, then the following statements are equivalent:

(i) $z=x+y, x \in C, y \in C^{-}$and $\langle x, y\rangle=0$;

(ii) $x=P_{C}(z)$ and $y=P_{C^{-}}(z)$, where $C^{-}$is the polar cone of $C$.

We now introduce the directional derivative of the operator $P_{K}$. 
Proposition 2.1. For any $x \in K$ and any element $v \in X$ the limit

$$
\Pi_{K}(x, v):=\lim _{\delta \rightarrow 0^{+}} \frac{P_{K}(x+\delta v)-x}{\delta}
$$

exists and $\Pi_{K}(x, v)=P_{T_{K}(x)}(v)$.

Proof. For a proof of this result we refer to [Z], Lemma 4.6, or [ $\underline{\mathrm{S}}$, Section 3.

Let $\Pi_{K}: K \times X \rightarrow X$ be the operator given by $(x, v) \mapsto \Pi_{K}(x, v)$. Note that whenever $v \in T_{K}(x)$, then $\Pi_{K}(x, v)=v$. The operator $\Pi_{K}$ is discontinuous on the boundary of the set $K$. Next we present a characterization of this operator.

Proposition 2.2. Let $X$ be a Hilbert space of arbitrary dimension and let $K \subset X$ be a non-empty, closed and convex subset. Then for each $x \in K$ there exists $n \in N_{K}(x)$ such that $\Pi_{K}(x, v)=v-n$, for any $v \in X$.

Proof. This is a direct consequence of Theorem 2.1 above.

We are now ready to define a projected differential equation.

Definition 2.2. Let $X$ be a Hilbert space of arbitrary dimension and let $K \subset X$ be a non-empty, closed and convex subset. Let $F: K \rightarrow X$ be a vector field. Then the ordinary differential equation

$$
\frac{d x(t)}{d t}=\Pi_{K}(x(t), F(x(t)))
$$

is called the projected differential equation associated with $F$ and $K$.

The projected differential equation is implied in $\mathrm{A}-\mathrm{C}$, Chapter 5 , Section 6 , in $[\mathrm{He}$ and in Is-C2, if one considers the case of single-valued mappings from $K$ to $X$. However, the first formulation of equation (1), in the form given above, occurred in 1990 in the paper $\left[\mathrm{D}-\mathrm{I}\right.$. There, $X=R^{n}$ and $K$ is a convex polyhedral set. This is then further used, in the same finite-dimensional context, in [Na2], [Na3], [Na4], [Na6], [Na5.

We now show that there is a fundamental relation between a projected differential equation and a variational inequality problem.

Definition 2.3. A point $x^{*} \in K$ is called a critical point for equation (11) if $\Pi_{K}\left(x^{*}, F\left(x^{*}\right)\right)=0$.

Theorem 2.2. Let $X$ be a Hilbert space of arbitrary dimension and let $K \subset X$ be a non-empty, closed and convex subset. Let $F: K \rightarrow X$ be a vector field. Consider the variational inequality problem:

$$
\text { find } x \in K \text { such that }\langle-F(x), y-x\rangle \geq 0, \forall y \in K \text {. }
$$

Then the solutions to the variational inequality problem coincide with the critical points of the projected differential equation (1).

Proof. The proof is immediate, based on Theorem 2.1 above.

For the dynamics of solutions to variational inequality problems in Hilbert spaces, we refer the reader to $\mathrm{Cj}]$, Is-C1] and [s-C2]. 


\section{Existence of solutions in Hilbert spaces}

We now consider a vector field $F: K \rightarrow X$. The solvability of the initial value problem

$$
\frac{d x(t)}{d t}=\Pi_{K}(x(t), F(x(t))), x(0)=x_{0} \in K
$$

was shown first in $\left[\mathrm{He}\right.$ in 1973, Section 2, for the case where $X=R^{n}, K \subset R^{n}$ a closed, convex subset and $F: K \rightarrow X$ an upper semicontinuous (see Definition 3.5 below) set-valued map with convex, compact values. The result appears again in A-C] in 1984, Chapter 5, Section 6, Theorem 1, in the same context. Another proof was given in [D-I] in 1990, Section 5.3, Theorem 5.1, and in [D-N] in 1993, Section 3.3 , Theorem 2, for the case where $X:=R^{n}, K \subset R^{n}$ a convex polyhedral set and $-F: X \rightarrow X$ satisfying $\|F(x)\| \leq B(1+\|x\|)$ and $\langle-F(x)+F(y), x-y\rangle \leq b\|x-y\|^{2}$. Finally, a proof for the existence of so-called slow solutions to problem (2) can be found in $\mathrm{Hi}$ in 1993, Chapter 2, Section 2, Theorem 2.2.2, for the case where $X:=R^{n}$ and $K:=R_{+}^{n}$. We show in this section, after several preliminary results, that problem (2) admits solutions in a suitable class of functions.

We now say what we mean by a solution to the initial value problem (2).

Definition 3.1. An absolutely continuous function $x: I \subset R \rightarrow X$ such that $x(t) \in K$ for all $t \in I$ and $\frac{d x(t)}{d t}=\Pi_{K}(x(t), F(x(t)))$, for almost all $t \in I$, is called a solution to the initial value problem (2).

We are now ready to state the main result of this paper.

Theorem 3.1. Let $X$ be a Hilbert space of arbitrary dimension and let $K \subset X$ be a non-empty, closed and convex subset. Let $F: K \rightarrow X$ be a Lipschitz continuous vector field with Lipschitz constant $b$. Let $x_{0} \in K$ and $L>0$ such that $\|x\| \leq L$.

Then the initial value problem $\frac{d x(t)}{d t}=\Pi_{K}(x(t), F(x(t))), x(0)=x_{0}$ has a unique solution on the interval $[0, l]$, where $l:=\frac{L}{\left\|F\left(x_{0}\right)\right\|+b L}$.

The proof of Theorem 3.1 is based on several technical results (which are going to be presented next) and uses elements from the theory of differential inclusions.

Under the hypothesis of Theorem 3.1, and inspired by Proposition 2.2, we can consider the projected differential equation (1) as a particular case of the differential inclusion $\frac{d x(t)}{d t} \in F(x(t))-N_{K}(x(t))$, for all $t \in[0, l]$. It is then natural to consider the following initial value problem:

$$
\frac{d x(t)}{d t} \in F(x(t))-N_{K}(x(t)), x(0)=x_{0} \in K
$$

Obviously, any solution to problem (2) is a solution to problem (3). In fact we have the following result ( $\mathrm{A}-\mathrm{C}$, Chapter 5, Section 6, Proposition 2):

Theorem 3.2. Any solution to problem (3) coincides with a solution to problem (2), and vice versa.

Now consider the set $\tilde{N}_{K}(x)=\left\{n \in N_{K}(x) \mid\|n\| \leq\|F(x)\|\right\} \subset N_{K}(x)$, for each $x \in K$. The set $\tilde{N}_{K}(x)$ is non-empty and for each $x \in K$ contains the unique element $n$ such that $\Pi_{K}(x, F(x))=F(x)-n$. 
Definition 3.2. An absolutely continuous function $x: I \subset R \rightarrow X$ such that $x(t) \in K$ for all $t \in I$ and $\frac{d x(t)}{d t} \in F(x(t))-\tilde{N}_{K}(x(t))$, for almost all $t \in I$, is called a solution to the initial value problem

$$
\frac{d x}{d t} \in F(x(t))-\tilde{N}_{K}(x(t)), x(0)=x_{0} \in K .
$$

Obviously, any solution to problem (4) is a solution to problem (3) and, in view of Theorem 3.2, is a solution to problem (2). Our aim now is to show that problem (44) has solutions.

Lemma 3.1. The set-valued mapping $x \mapsto F(x)-\tilde{N}_{K}(x)$ has non-empty, closed and convex values for each $x \in K$.

Proof. The proof is immediate from the construction of the set $\tilde{N}_{K}(x)$.

Definition 3.3. For any subset $D \subset X$, the function $\sigma_{D}: X \rightarrow(-\infty,+\infty]$, given by $\sigma_{D}(p):=\sup _{y \in D}\langle p, y\rangle$, is called the support function of $D$.

For convenience we write $\sigma_{D}(p)=\sigma(D, p)$. The support functions characterize any non-empty closed and convex set $K \subset X$ by $K:=\{y \in Y \mid \forall p \in X,\langle p, y\rangle \leq$ $\sigma(K, p)\}$. Let $E$ and $F$ be two Hausdorff topological vector spaces, let $f: R \rightarrow R$ be a function and let $G: E \rightarrow 2^{F}$ be a set-valued mapping.

Definition 3.4. The function $f$ is upper semicontinuous (as a single-valued function) at $x \in R$ if for all $\epsilon>0$, there exists $\delta>0$ such that $|y-x|<\delta \Longrightarrow$ $f(y) \leq f(x)+\epsilon$.

Definition 3.5. The mapping $G$ is called upper semicontinuous at $x_{1} \in E$ (u.s.c.) if for each open neighbourhood $V$ of $G\left(x_{1}\right)$, there exists a neighbourhood $U$ of $x_{1}$ such that $G(U) \subset V$. $G$ is called upper semicontinuous if it is so at each $x_{1} \in E$.

Assume we are given a set-valued map $G$ from $K$ into $X$. For every $p \in X$, we can consider the function from $K$ to $(-\infty,+\infty]$, given by $x \mapsto \sigma(G(x), p)$.

Definition 3.6. We say that $G$ is upper hemicontinuous at $x_{1} \in K$ (u.h.c.) if, for every $p \in X$, the function $x \mapsto \sigma(G(x), p)$ is upper semicontinuous as a single-valued function at $x_{1}$. $G$ is called upper hemicontinuous if it is u.h.c. at each $x_{1} \in K$.

Proposition 3.1. Let $X$ be a Hilbert space of arbitrary dimension and $K \subset X a$ non-empty, closed and convex subset. Let $F: K \rightarrow X$ be a Lipschitz continuous vector field with Lipschitz constant $b$, so that on $K \cap B\left(x_{0}, L\right)$, with $L>0$ and $x_{0} \in K$ arbitrarily fixed, we have $\|F(x)\| \leq M:=\left\|F\left(x_{0}\right)\right\|+b L$. Then the setvalued mapping $\mathcal{N}_{p}: K \cap B\left(x_{0}, L\right) \rightarrow R$ given by $x \mapsto\left\langle-\tilde{N}_{K}(x), p\right\rangle$ has a closed graph.

Proof. It is obvious that for each $p \in X$, the set-valued map $\mathcal{N}_{p}: K \cap B\left(x_{0}, L\right) \rightarrow R$ maps $K \cap B\left(x_{0}, L\right)$ into $2^{[-M\|p\|, M\|p\|]}$. Let $\left\{\left(x_{n}, z_{n}\right)\right\}_{n} \in \operatorname{graph}\left(\mathcal{N}_{p}\right)$ and such that $\left(x_{n}, z_{n}\right) \rightarrow(x, z)$, where $(x, z) \in X \times 2^{[-M\|p\|, M \| p||]}$. We want to show that $(x, z) \in \operatorname{graph}\left(\mathcal{N}_{p}\right)$. From $z_{n} \in \mathcal{N}_{p}\left(x_{n}\right)$, for all $n$, we deduce that $z_{n}:=\left\langle y_{n}, p\right\rangle$, with $y_{n} \in-\tilde{N}_{K}\left(x_{n}\right)$. Since the set $-\tilde{N}_{K}\left(x_{n}\right) \subset \overline{B(0, M)}$ and $\overline{B(0, M)}$ is weakly 
compact, then there exists $y \in X$ such that $\left\langle\beta, y_{n}\right\rangle \rightarrow\langle\beta, y\rangle$ for all $\beta \in X$ and a suitable subsequence of $\left\{y_{n}\right\}$, denoted by $\left\{y_{n}\right\}$ as well.

Suppose $y \notin-\tilde{N}_{K}(x):=\{n \mid\langle w-x, n\rangle \geq 0, \forall w \in K$ and $\|n\| \leq\|F(x)\| \leq M\}$. Then there exists $w \in K$ such that $\langle w-x, y\rangle\left\langle\lambda<0\right.$. Since $\left\langle\beta, y_{n}\right\rangle \rightarrow\langle\beta, y\rangle$ for all $\beta \in X$ this implies that for sufficiently large $n,\left\langle w-x, y_{n}\right\rangle<\frac{\lambda}{2}<0$. But: $\left\langle w-x_{n}, y_{n}\right\rangle=\left\langle w-x, y_{n}\right\rangle+\left\langle x-x_{n}, y_{n}\right\rangle$ and since $x_{n} \rightarrow x$, there exists $n_{0}>0$ such that $\forall n \geq n_{0}$, we have $\left\langle x-x_{n}, y_{n}\right\rangle \leq\left\|x-x_{n}\right\| \cdot\left\|y_{n}\right\|<\frac{|\lambda|}{4 M} M=\frac{|\lambda|}{4}$. Thus $\left\langle w-x_{n}, y_{n}\right\rangle<\frac{\lambda}{4}<0$, for all $n \geq n_{0}$. But this contradicts the fact that $y_{n} \in-\tilde{N}_{K}\left(x_{n}\right)$. This proves $y \in-\tilde{N}_{K}(x)$ and therefore $(x, z) \in \operatorname{graph}\left(\mathcal{N}_{p}\right)$.

Proposition 3.2. Let $X$ be a Hilbert space of arbitrary dimension, let $K \subset X$ be a non-empty closed and convex subset and let $F: K \rightarrow X$ be as in Proposition 3.1. Then, for each $p \in X$, the set-valued mapping $x \mapsto\left\langle F(x)-\tilde{N}_{K}(x), p\right\rangle$ has a closed graph.

Proof. This proof is immediate, based on Proposition 3.1 above.

Theorem 3.3. Let $X$ be a Hilbert space of arbitrary dimension and let $K \subset X$ be a non-empty closed, convex subset. Let $F: K \rightarrow X$ be a Lipschitz continuous vector field so that on $K \cap B\left(x_{0}, L\right)$, with $L>0$ and $x_{0} \in K$, we have $\|F(x)\| \leq M:=$ $\left\|F\left(x_{0}\right)\right\|+b L$. Let $l:=\frac{L}{M}$ and $I:=[0, l]$. Then there exists a sequence $\left\{x_{k}(\cdot)\right\}$ of absolutely continuous functions defined on $I$, with values in $K$, such that for all $k \geq 0, x_{k}(0)=x_{0}$ and for almost all $t \in I,\left\{x_{k}(t)\right\}$ and $\left\{x_{k}^{\prime}(t)\right\}$ (the sequence of its derivatives) have the following property: for every neighbourhood $\mathcal{M}$ of 0 in $X \times X$, there exists $k_{0}=k_{0}(t, \mathcal{M})$ such that

$$
\forall k \geq k_{0},\left(x_{k}(t), x_{k}^{\prime}(t)\right) \in \operatorname{graph}\left(F-\tilde{N}_{K}\right)+\mathcal{M} .
$$

Proof. Let $r>0$ and $y \in K$ be arbitrarily fixed. Since $\frac{P_{K}(y+h F(y))-y}{h} \rightarrow$ $\Pi_{K}(y, F(y))$ as $h \rightarrow 0^{+}$, we deduce that we can choose a scalar $0<h_{y}<r$ such that

$$
\left\|\Pi_{K}(y, F(y))-\frac{P_{K}\left(y+h_{y} F(y)\right)-y}{h_{y}}\right\|<\frac{r}{2} .
$$

By the continuity of the mapping $P_{K}\left(i d_{K}+h_{y} F\right)-i d_{K}$ we have that there exists $\rho \in(0, r)$ such that for all $x \in B(y, \rho) \cap K$,

$$
\left\|\frac{P_{K}\left(y+h_{y} F(y)\right)-y}{h_{y}}-\frac{P_{K}\left(x+h_{y} F(x)\right)-x}{h_{y}}\right\|<\frac{r}{2} .
$$

Combining these we have that for any $y \in X$, there exist $0<h_{y}<r$ and $0<\rho<r$ such that $\forall x \in B(y, \rho) \cap K$, there exists $z_{y}:=P_{K}\left(x+h_{y} F(x)\right) \in K$ with the property

$$
\left\|\Pi_{K}(y, F(y))-u\right\|<r, \text { where } u:=\frac{z_{y}-x}{h_{y}} .
$$

Applying the preceding discussion with $y=x_{0}$, we see that there exist scalar $0<h_{0}<r$ and $0<\rho_{0}<r$ such that $\forall x \in B\left(x_{0}, \rho_{0}\right) \cap K$, there exist $z_{0} \in K$ and $u_{0}:=\frac{z_{0}-x}{h_{0}}$ with the properties

- $x_{0}+h^{0} u^{0} \in K$,

- $\left(x_{0}, u^{0}\right) \in \operatorname{graph}\left(F-\tilde{N}_{K}\right)+r(B \times B)$, where $B$ is the open unit ball of $X$,

- $h_{0} \geq \frac{1}{2} \sup h$, where the sup is taken over all pairs $(h, u)$ satisfying these conditions. 
Now let $x^{1}:=x_{0}+h^{0} u^{0} \in K$. Then there exist $h_{1}<r$ and $0<\rho_{1}<r$ such that $\forall x \in B\left(x^{1}, \rho_{1}\right) \cap K$, there exist $z_{1}:=P_{K}\left(x+h_{1} F(x)\right) \in K$ and $u_{1}:=\frac{z_{1}-x}{h_{1}}$ with the properties

- $x^{1}+h^{1} u^{1} \in K$,

- $\left(x^{1}, u^{1}\right) \in \operatorname{graph}\left(F-\tilde{N}_{K}\right)+r(B \times B)$,

- $h_{1} \geq \frac{1}{2} \sup h$, where the sup is taken over all pairs $(h, u)$ satisfying these conditions.

Now let $x^{2}:=x^{1}+h^{1} u^{1} \in K$. Continuing this construction, we obtain sequences of $x^{p}$ 's and $u^{p}$ 's, such that $x^{p+1}:=x^{p}+h^{p} u^{p}$ and $\left(x^{p}, u^{p}\right) \in \operatorname{graph}\left(F-\tilde{N}^{K}\right)+r(B \times B)$. We note that if the sequence of scalars $h^{p} \rightarrow 0$, the sequence $\left\{x^{p}\right\}$ is Cauchy, for then $\left\|x^{p+1}-x^{p}\right\| \leq h^{p}\left\|F\left(x^{p}\right)\right\| \rightarrow 0$. This means that $x^{p}$ has a limit in $X$, say $x^{*}$. Then for any neighbourhood $U$ of the limit $x^{*}$, there exists $p^{0}>0$ such that $\forall p>p^{0}, x^{p} \in U$. In particular, if $U=B\left(x^{*}, \rho^{*}\right)$, then for $p>p^{0}, h^{p} \geq \frac{1}{2} h^{*}$. This proves that we cannot have $h^{p} \rightarrow 0$. That is, we can be sure that there exists an index $m>0$ so that $h^{0}+h^{1}+\ldots+h^{m} \leq l \leq h^{1}+\ldots+h^{m+1}$.

We now apply this construction with a succession of values of $r$, thereby creating a sequence of piecewise continuous approximate solutions to the projected differential equation. With $r:=\frac{1}{k}$ we let $\tau_{k}^{q}=h^{0}+\ldots+h^{q-1}$, and $\tau_{k}^{0}=0$. Evidently, $\tau_{k}^{q}-\tau_{k}^{q-1}=h^{q-1}<\frac{1}{k}$.

We parametrize the curve by interpolating $x_{k}(t):=x^{p-1}+\left(t-\tau_{k}^{p-1}\right) u^{p-1}$. Let $t \in\left(\tau_{k}^{p-1}, \tau_{k}^{p}\right)$ be fixed. We claim that there exists a pair $(z, v) \in \operatorname{graph}\left(F-\tilde{N}_{K}\right)$ such that $\left\|x_{k}(t)-z\right\| \leq \epsilon_{k}$ and $\left\|x_{k}^{\prime}(t)-v\right\| \leq \epsilon_{k}$ where $\epsilon_{k} \rightarrow 0$ when $k \rightarrow \infty$. The existence of the pair $(z, v)$ with the claimed properties is a consequence of the construction of the sequence $\left\{x_{k}\right\}$. We have $\left(x^{p-1}, u^{p-1}\right) \in \operatorname{graph}\left(F-\tilde{N}_{K}\right)+$ $\frac{1}{k}(B \times B)$. That is, there exists $(z, v) \in \operatorname{graph}\left(F-\tilde{N}_{K}\right)$ such that $\left\|z-x^{p-1}\right\|<\frac{1}{k}$ and $\left\|u^{p-1}-v\right\|<\frac{1}{k}$. Therefore

$$
\begin{aligned}
\left\|x_{k}(t)-z\right\| & \leq\left\|x_{k}(t)-x^{p-1}\right\|+\left\|x^{p-1}-z\right\| \leq\left|t-\tau_{k}^{p-1}\right| \cdot\left\|u^{p-1}\right\|+\frac{1}{k} \\
& \leq \frac{1}{k}\left(\left\|F\left(x^{p-1}\right)\right\|+1\right) \leq \frac{1}{k}(M+1)=: \epsilon_{k}
\end{aligned}
$$

and $\left\|x_{k}^{\prime}(t)-v\right\|=\left\|u^{p-1}-v\right\|<\frac{1}{k}$. In conclusion, we have $\left(x_{k}(t), x_{k}^{\prime}(t)\right) \in$ $\operatorname{graph}\left(F-\tilde{N}_{K}\right)+\epsilon_{k}(B \times B)$ where $\epsilon_{k} \rightarrow 0$, when $k \rightarrow \infty$.

Absolutely continuous functions with values in a Hilbert space $X$ can be characterized as follows.

Theorem 3.4. If $f, g: I:=[0, l] \rightarrow X$ and $t_{0} \in I$, then the following conditions are equivalent:

(i) $f$ is absolutely continuous and a.e. differentiable and $f^{\prime}(t)=g(t)$, for a.a. $t \in I$.

(ii) $g$ is Bochner integrable and $f(t)=f\left(t_{0}\right)+\int_{t_{0}}^{t} g(s) d s$, for all $t \in I$.

Proof. The proof can be found in [Hk], Chapter 6, Section 4, Theorem 1.4.6.

For a brief account of Bochner integrability, as well as the relationship between the spaces $L^{1}(I, X)$ and $L^{\infty}(I, X)$, which will be used in the following, we refer 
the reader to $[\mathrm{Sw}$, Chapter 26, Section 3, and [D-S], Part I, Chapter IV, Section 8. Note especially that $L^{\infty}(I, X)$ is the dual of the space $L^{1}(I, X)$ (see [D-S], Part I, Chapter IV, Section 8, Theorem 5). We consider the Lebesgue measure on $I$. We are now ready to prove the main result of this paper, namely Theorem 3.1. We do this by showing that the sequence of approximate solutions constructed in Theorem 3.3 has a subsequence that converges uniformly to an absolutely continuous curve and that this limit curve is a solution to the differential inclusion (4). A simple argument at the end of the proof will establish uniqueness.

Proof of Theorem 3.1. (i) We begin by proving the uniform convergence of the sequence $\left\{x_{k}(\cdot)\right\}$ constructed in Theorem 3.3. By Theorem 3.3 we know that there exists a pair $\left(u_{k}(t), F\left(u_{k}(t)\right)-n_{k}\right) \in \operatorname{graph}\left(-F-\tilde{N}_{K}\right)$ such that $x_{k}(t)-u_{k}(t)=\epsilon_{1, k}(t)$ and $x_{k}^{\prime}(t)-F\left(u_{k}(t)\right)+n_{k}=\epsilon_{2, k}(t)$, where $\epsilon_{1, k}(t)$ and $\epsilon_{2, k}(t)$ are vector functions, not necessarily continuous, satisfying $\left\|\epsilon_{1, k}(t)\right\|<\epsilon_{k}$ and $\left\|\epsilon_{2, k}(t)\right\|<\epsilon_{k}$ where $\epsilon_{k} \rightarrow 0$ as $k \rightarrow \infty$. Let $k, m$ be two indexes. Then we evaluate $\frac{1}{2} \frac{d}{d t}\left\|x_{k}(t)-x_{m}(t)\right\|^{2}=$ $\left\langle x_{k}^{\prime}(t)-x_{m}^{\prime}(t), x_{k}(t)-x_{m}(t)\right\rangle=\left\langle F\left(u_{k}(t)\right)-n_{k}-F\left(u_{m}(t)\right)+n_{m}, x_{k}(t)-x_{m}(t)\right\rangle+$ $\left\langle\epsilon_{2, k}(t)-\epsilon_{2, m}(t), x_{k}(t)-x_{m}(t)\right\rangle$, where $n_{k} \in \tilde{N}_{K}\left(u_{k}(t)\right)$ and $n_{m} \in \tilde{N}_{K}\left(u_{m}(t)\right)$. Then

$$
\begin{aligned}
\frac{1}{2} \frac{d}{d t}\left\|x_{k}(t)-x_{m}(t)\right\|^{2}= & \left\langle F\left(u_{k}(t)\right)-F\left(x_{k}(t)\right)-F\left(u_{m}(t)\right)+F\left(x_{m}(t)\right), x_{k}(t)-x_{m}(t)\right\rangle \\
& +\left\langle F\left(x_{k}(t)\right)-F\left(x_{m}(t)\right), x_{k}(t)-x_{m}(t)\right\rangle \\
& +\left\langle-n_{k}+n_{m}, u_{k}(t)-u_{m}(t)\right\rangle \\
& +\left\langle-n_{k}+n_{m},-u_{k}(t)+x_{k}(t)+u_{m}(t)-x_{m}(t)\right\rangle \\
& +\left\langle\epsilon_{2, k}(t)-\epsilon_{2, m}(t), x_{k}(t)-x_{m}(t)\right\rangle \\
\leq & \left\langle F\left(x_{k}(t)\right)-F\left(x_{m}(t)\right), x_{k}(t)-x_{m}(t)\right\rangle+\left(\epsilon_{k}+\epsilon_{m}\right)\left\|n_{k}-n_{m}\right\| \\
& +b\left(\epsilon_{k}+\epsilon_{m}\right)\left\|x_{k}(t)-x_{m}(t)\right\|+\left(\epsilon_{k}+\epsilon_{m}\right)\left\|x_{k}(t)-x_{m}(t)\right\|,
\end{aligned}
$$

where to obtain the last inequality we used the fact that $x \mapsto \tilde{N}_{K}(x)$ is a monotone set-valued map (see Lemma 2.1) and the Lipschitz continuity of $F$.

We now let $\phi(t):=\left\|x_{k}(t)-x_{m}(t)\right\|$. Then we obtain from above that

$$
\phi^{\prime}(t) \phi(t) \leq b \phi(t)^{2}+\left(\epsilon_{k}+\epsilon_{m}\right)[(1+b) \phi(t)+2 M] .
$$

But $\phi(t) \leq 2 L$, so $\phi^{\prime}(t) \phi(t) \leq b \phi(t)^{2}+\left(\epsilon_{k}+\epsilon_{m}\right)[(1+b) 2 L+2 M]$. Let $a:=$ $(1+b) 2 L+2 M$. Then $\phi^{\prime}(t) \phi(t)-b \phi(t)^{2} \leq a\left(\epsilon_{k}+\epsilon_{m}\right)$. We have the following evaluation: $\frac{d}{d t}\left[\phi(t)^{2} e^{-2 b t}\right]=2 e^{-2 b t}\left[\phi^{\prime}(t) \phi(t)^{2}-2 b \phi(t)^{2}\right]$. Using the last two relations together we obtain $\frac{d}{d t}\left[\phi(t)^{2} e^{-2 b t}\right] \leq 2 e^{-2 b t} a\left(\epsilon_{k}+\epsilon_{m}\right)$. Now by integration between 0 and $t$ we further obtain $\phi(t)^{2} e^{-2 b t} \leq \frac{a}{b}\left(\epsilon_{k}-\epsilon_{m}\right)\left(1-e^{-2 b t}\right)$. Multiplying the last inequality with $e^{2 b t}$ we get

$$
\phi(t)^{2} \leq \frac{a}{b}\left(\epsilon_{k}+\epsilon_{m}\right)\left(e^{2 b t}-1\right) \leq \frac{a}{b}\left(\epsilon_{k}+\epsilon_{m}\right)\left(e^{2 b l}-1\right),
$$

where $l$ is the length of $I$. That is, the sequence $\left\{x_{k}(\cdot)\right\}$ converges uniformly on $I$.

(ii) Having proved the uniform convergence of the sequence $x_{k}(\cdot)$ of approximate solutions to a limit $x(\cdot)$, we will now select a subsequence whose derivatives con- 
verge weakly* in $L^{\infty}(I, X)$ to its derivative $x^{\prime}(\cdot)$. Since $\left\|x_{k}^{\prime}(t)\right\| \leq C$ for some fixed $C$, we have $x_{k}^{\prime}(t) \in \bar{B}(0, C) \subset L^{\infty}(I, X)$ which is weakly* compact by Alaoglu's Theorem (see [A-C], Chapter 0, Section 3, Theorem 3) and the fact that $L^{\infty}(I, X)$ is the dual of $L^{1}(I, X)$. Thus, there exists a subsequence of $\left\{x_{k}^{\prime}(\cdot)\right\}$, again denoted by $\left\{x_{k}^{\prime}(\cdot)\right\}$, which converges weakly* to $w(\cdot)$ in $L^{\infty}(I, X)$. This means that for every $\alpha(\cdot) \in L^{1}(I, X), \int_{I}\left\langle\alpha(\tau), x_{k}^{\prime}(\tau)\right\rangle d \tau \rightarrow \int_{I}\langle\alpha(\tau), w(\tau)\rangle d \tau$. In particular, letting $\alpha(\tau)=\chi_{[s, t]}(\tau) \alpha$ for $\alpha \in X$ (where by $\chi_{[s, t]}$ we denoted the characteristic function of the interval $[s, t])$ we have $\int_{s}^{t}\left\langle\alpha, x_{k}^{\prime}(\tau)\right\rangle d \tau \rightarrow \int_{s}^{t}\langle\alpha, w(\tau)\rangle d \tau$. Since $\left\{x_{k}(\cdot)\right\}$ is absolutely continuous we also have $\left\langle\alpha, x_{k}(t)-x_{k}(s)\right\rangle=\int_{s}^{t}\left\langle\alpha, x_{k}^{\prime}(\tau)\right\rangle d \tau$. Taking limits as $k \rightarrow \infty$, we conclude that $\langle\alpha, x(t)-x(s)\rangle=\int_{s}^{t}\langle\alpha, w(\tau)\rangle d \tau=\left\langle\alpha, \int_{s}^{t} x^{\prime}(\tau) d \tau\right\rangle$. Since this is true for every $\alpha \in X$, it follows that $x(t)-x(s)=\int_{s}^{t} w(\tau) d \tau$. By Theorem 3.4 this implies that $x(\cdot)$ is absolutely continuous and $x^{\prime}(\cdot)=w(\cdot)$.

(iii) We will now use Proposition 3.2 to show that $x(\cdot)$ is a solution to the differential inclusion (4).

Once again we use the fact that for each $k \geq k_{0}$ and almost every $t \in I$ there exists a pair $\left(u_{k}(t), v_{k}(t)\right) \in \operatorname{graph}\left(F-\tilde{N}_{K}\right)$ such that $\left\|x_{k}(t)-u_{k}(t)\right\|<\epsilon_{k}$ and $\left\|x_{k}^{\prime}(t)-v_{k}(t)\right\|<\epsilon_{k}$, where $\epsilon_{k} \rightarrow 0$ when $k \rightarrow \infty$. Let $p \in X$ be arbitrarily fixed. Then for almost all $t,\left(u_{k}(t),\left\langle p, v_{k}(t)\right\rangle\right) \in \operatorname{graph}\left(\left\langle p, F-\tilde{N}_{K}\right\rangle\right)$ and $\|\left\langle p, x_{k}^{\prime}(t)\right\rangle-$ $\left\langle p, v_{k}(t)\right\rangle\|<\| p \| \epsilon_{k}$. Evidently, $u_{k}(t)$ converges to $x(t)$ for every $t \in I$ and $\left\langle p, v_{k}(t)\right\rangle$ converges to $\left\langle p, x^{\prime}(t)\right\rangle$ for almost all $t \in I$.

Then, by Proposition 3.2, $\operatorname{graph}\left(\left\langle p, F-\tilde{N}_{K}\right\rangle\right)$ is closed, so by letting $k \rightarrow \infty$ it follows that for almost all $t,\left(x(t),\left\langle p, x^{\prime}(t)\right\rangle\right) \in \operatorname{graph}\left(\left\langle p, F-\tilde{N}_{K}\right\rangle\right)$. Since the set $F(x(t))-\tilde{N}_{K}(x(t))$ is convex and closed it follows that $x^{\prime}(t)$ is a solution to problem (4), and therefore to problem (2).

(iv) Finally, we are able to show easily the uniqueness of solutions to problem (2). From (i)-(iii) above we have that the problem has solutions on an interval $I:=[0, l]$. Suppose $x_{1}(\cdot)$ and $x_{2}(\cdot)$ are two solutions starting at the point $x_{0}$. Then for any fixed $t \in I$,

$$
\begin{aligned}
\frac{d}{d t}\left(\frac{1}{2}\right. & \left.\left\|x_{1}(t)-x_{2}(t)\right\|^{2}\right) \\
& =\left\langle x_{1}(t)-x_{2}(t), \Pi_{K}\left(x_{1}(t), F\left(x_{1}(t)\right)\right)-\Pi_{K}\left(x_{2}(t), F\left(x_{2}(t)\right)\right)\right\rangle \\
& \leq b\left\|x_{1}(t)-x_{2}(t)\right\|^{2} .
\end{aligned}
$$

Applying Gronwall's inequality for the function $t \rightarrow\left\|x_{1}(t)-x_{2}(t)\right\|^{2}$ we obtain $\left\|x_{1}(t)-x_{2}(t)\right\|^{2} \leq 0$, i.e., $x_{1}(t)=x_{2}(t)$ for any $t \in I$.

\section{Concluding Remarks}

The study of the dynamic properties of solutions to infinite-dimensional equilibrium problems through a projected dynamics has been started in [s-C2] and Cj], inspired by the technique introduced in [D-I], $[\mathrm{D}-\mathrm{N}]$ and [Na2] in the finitedimensional case.

We would like to thank the referee for valuable comments and observations which have led to a clearer presentation of our result. 


\section{REFERENCES}

[A-C] AUBIN, J. P. and CELLINA, A., Differential Inclusions, Set-valued maps and viability theory, Grundlehren der mathematischen Wissenschaften, Vol. 264, Springer-Verlag, Berlin (1984). MR 85j:49010

[B-C] BAIOCCHI, C. and CAPELO, A., Variational and Quasivariational Inequalities. Applications to free boundary problems, J. Wiley and Sons, 1984. MR 86e:49018

[Cj] COJOCARU, M. G., Projected Dynamical Systems on Hilbert Spaces, Ph.D. Thesis, Queen's University, 2002.

[D-S] DUNFORD, N. and SCHWARTZ, J. T., Linear Operators, Wiley Classics Library Ed., New York: Wiley-Interscience (1988). MR 90g:47001a. MR 90g:47001b MR 90g:47001c

[D-I] DUPUIS, P. and ISHII, H., On Lipschitz continuity of the solution mapping to the Skorokhod problem, with applications, Stochastics and Stochastics Reports, Vol. 35(1990), 31-62. MR 93e:60110

[D-N] DUPUIS, P. and NAGURNEY, A., Dynamical systems and variational inequalities, Annals of Operations Research 44, (1993), 9-42. MR 94k:49009

[He] HENRY, C., An existence theorem for a class of differential equations with multivalued right-hand sides, J. Math. Anal. Appl. 41 (1973), 179-186. MR 49:684

[Hk] HEIKKILA, S., Monotonone Iterative Techniques for Discontinuous Nonlinear Differential Equations, Monographs and Textbooks in Pure and Applied Mathematics, Vol. 181, Marcel Dekker (1994). MR 95d:34002

[Hi] HIPFEL, D., The Nonlinear Differential Complementarity Problem, Ph. D. Thesis, Rensselaer Polytechnic Institute (1993).

[Ra] HYERS, D. H., ISAC G. and RASSIAS, Th. M., Topics in Nonlinear Analysis and Applications, World Scientific Publ. Co., River Edge, NJ, 1997. MR 98g:47001

[Is] ISAC, G., Complementarity Problems, Lecture Notes in Mathematics, No. 1528, Springer-Verlag, Berlin, 1992. MR 94h:49002

[Is-C1] ISAC, G. and COJOCARU, M. G., The projection operator in a Hilbert space and its directional derivative. Consequences for the theory of projected dynamical systems, preprint, 2002.

[Is-C2] ISAC, G. and COJOCARU, M. G., Variational inequalities, complementarity problems and pseudo-monotonicity. Dynamical aspects, in "Seminar on fixed point theory ClujNapoca" (Proceedings of the International Conference on Nonlinear Operators, Differential Equations and Applications, September 2002, Romania), Babes-Bolyai University of Cluj-Napoca, Vol. III (2002), 41-62.

[K-S] KINDERLEHRER, D. and STAMPACCHIA, G., An Introduction to Variational Inequalities and Their Applications, Pure and Applied Math., Vol. 88, Academic Press, 1980. MR 81g:49013

[Na1] NAGURNEY, A., Network Economics. A Variational Inequality Approach, Kluwer Academic Publishers, Dordrecht, 1993. MR 93m:90002

[Na2] NAGURneY, A. and ZHANG, D., Projected Dynamical Systems and Variational Inequalities with Applications, Kluwer Academic Publishers (1996).

[Na3] NAGURNEY, A. and ZHANG, D., On the stability of an adjustment process for spatial price equilibrium modeled as a projected dynamical system, Journal of Economic Dynamics and Control 20, (1996), 43-63. MR 96j:90021

[Na4] NAGURNEY, A., DUPUIS P. and ZHANG, D., A dynamical systems approach for network oligopolies and variational inequalities, Annals of Regional Science 28, (1994), 263283.

[Na5] NAGURneY, A. and SIOKOS, S., Financial Networks: Statics and Dynamics, Springer-Verlag, New York, 1997.

[Na6] NAGURneY, A., TAKAYAMA, T. and ZHANG, D., Projected dynamical systems, modeling and computation of spatial network equilibria, Networks 26, (1995), 69-85.

[P] PAPPALARDO, M. and PASSACANTANDO, M., Stability for equilibrium problems: from variational inequalities to dynamical systems, J. Opt. Theory Appl. 113, (2002), 567-582.

[S] SHAPIRO, A. S., Existence and differentiability of metric projections in Hilbert spaces, SIAM J. Optimization 4 (1994), 130-141. MR 94m:90111 
[Sw] SWARTZ, C., An Introduction to Functional Analysis, Pure and Applied Mathematics Series, 154, Marcel Dekker (1992). MR 93c:46002

[Z] ZARANTONELlO, E., Projections on convex sets in Hilbert space and spectral theory, Contributions to Nonlinear Functional Analysis, Publ. No. 27, Proc. Sympos. Math. Res. Center, Univ. Wisconsin, Academic Press (1971), 237-424. MR 52:9014

Department of Mathematics and Statistics, Jeffery Hall, Room 207, Queen's UniverSity, Kingston, Ontario, Canada K7M 2W8

E-mail address: monica@mast.queensu.ca

Current address: Department of Mathematics and Statistics, Room 536 MacNaughton Building, University of Guelph, Guelph, Ontario, Canada N1G 2W1

Department of Mathematics and Statistics, Jeffery Hall, Room 508, Queen's UniverSity, Kingston, Ontario, Canada K7M 2W8

E-mail address: leo@mast.queensu.ca 